\title{
Puromycin-sensitive aminopeptidase is involved in wild-type huntingtin clearance
}

\author{
Guijie Ren ${ }^{1,2}$, Zonghcai Ma', Maria Hui ${ }^{3}$, Lili C Kudo ${ }^{4}$, Koon-Sea Hui ${ }^{3}$, Stanislav L Karsten ${ }^{1,4,5^{*}}$ \\ From 2011 International Conference on Molecular Neurodegeneration \\ Shanghai, China. 22-24 September 2011
}

\begin{abstract}
Background
Accumulation of neurotoxic mutated huntingtin protein (Htt) is an important contributing factor to the pathogenesis of Huntington's disease (HD). More and more evidence has showed that wild-type Htt plays important roles in maintaining normal functions of neuronal system. The clearance of mutant $\mathrm{Htt}$ has been studied extensively; however, little is known about the mechanisms regulating accumulation and turnover of endogenous wild-type $\mathrm{Htt}$. Puromycin-sensitive aminopeptidase (PSA) was recently identified as a major peptidase digesting TAU protein and protecting against TAU-induced neurodegeneration. PSA was also found as the peptidase responsible for digesting polyglutamine sequences released by proteasomes and removal of neurotoxic polyglutamine-expanded Htt exon1 , ataxin-3, mutant synuclein and superoxide dismutase 1 via autophagy system. These results suggest that PSA might represent a novel degradation mechanism targeting aggregate-prone neurotoxic protein substrates, including mutated Htt. However, the effects of PSA on endogenous wild-type $\mathrm{Htt}$ abundance remain unclear. Here, we investigated the effects of PSA on endogenous wild-type $\mathrm{Htt}$ clearance in vivo using a PSA overexpressing transgenic murine model and in vitro using human neuroblastoma cell line.
\end{abstract}

\section{Methods}

We first determined whether PSA regulates Htt abundance in vivo using a human PSA (hPSA) transgenic mouse model, which was generated using bacterial artificial chromosome (BAC) mediated technology. For measuring the Htt expression in hPSA transgenic mice and control mice, the brain tissues were dissected and separated, and then

\footnotetext{
* Correspondence: skarsten@neuroindx.com

'Division of Neuroscience, Los Angeles Biomedical Research Institute at Harbor-UCLA Medical Center, Torrance, CA 90502, USA

Full list of author information is available at the end of the article
}

were homogenized by sonication followed with centrifugation at $100,000 \mathrm{~g}$ at $4^{\circ} \mathrm{C}$. Htt in supernatants were analyzed by Western blot using anti-Htt antibodies. Immunohistochemistry was also performed using perfused brains from both hPSA and control mice according to standard protocols. And we tested whether PSA overexpression is able to reduce the abundance of endogenous $\mathrm{Htt}$ in human neuroblastoma cells SH-SY5Y in vitro. SH-SY5Y cells were transfected with hPSA overexpression vectors pCMV6-XLhPSA or blank vectors and then the endogenous Htt was analyzed with Western blot and immunocytochemistry. We also investigated the effect of inhibition of PSA expression using RNA interference (Stealth-RNAi) on endogenous Htt levels in SH-SY5Y cells. Cells were transfected with siRNA oligonucleotides specific for hPSA. Efficient hPSA silencing was confirmed with both real-time RTPCR and Western blot analysis.

\section{Results}

In vivo studies, Western blot analysis showed that $\mathrm{Htt}$ is significantly decreased in human PSA transgenic mice. Immunohistochemical experiment also showed $\mathrm{Htt}$ expression is decreased in all the transgenic brain regions tested. In vitro studies, Western blot analysis demonstrated decrease of endogenous $\mathrm{Htt}$ in response to PSA overexpression and accumulation of $\mathrm{Htt}$ in response to PSA slicing with Stealth-RNAi in human neuroblastoma cell line SH-SY5Y. And immunocytochemistry demonstrated notable decrease and rapid accumulation of $\mathrm{Htt}$ in response to PSA overexpression and PSA slicing respectively in SH-SY5Y cells.

\section{Conclusion}

These combined in vivo and in vitro data implicate that PSA may be a key physiological regulator of clearance and turnover of wild-type Htt in neuronal system, and may play important roles in pathogenesis of HD through 
regulating clearance of wild-type or and mutated $\mathrm{Htt}$ as well.

\section{Author details}

'Division of Neuroscience, Los Angeles Biomedical Research Institute at Harbor-UCLA Medical Center, Torrance, CA 90502, USA. ${ }^{2}$ Department of Biochemistry and Molecular Biology, Medical College, Shandong University, Jinan, Shandong, 250012, China. ${ }^{3}$ Nathan S. Kline Institute for Psychiatric Research, New York University School of Medicine, Orangeburg, NY 10962, USA. ${ }^{4}$ NeurolnDx Inc., 1655 East 28th Street, Signal Hill, CA 90755, USA. ${ }^{5}$ Department of Neurology, David Geffen School of Medicine at UCLA, Los Angeles, CA 90095, USA.

Published: 7 February 2012

doi:10.1186/1750-1326-7-S1-S5

Cite this article as: Ren et al:: Puromycin-sensitive aminopeptidase is involved in wild-type huntingtin clearance. Molecular Neurodegeneration 2012 7(Suppl 1):S5.

Submit your next manuscript to BioMed Central and take full advantage of:

- Convenient online submission

- Thorough peer review

- No space constraints or color figure charges

- Immediate publication on acceptance

- Inclusion in PubMed, CAS, Scopus and Google Scholar

- Research which is freely available for redistribution

Submit your manuscript at www.biomedcentral.com/submit 